\title{
Polyaniline deposition on tilted fiber Bragg grating for $\mathrm{pH}$ sensing
}

\author{
A. Lopez Aldaba*a,b, Á. González-Vila ${ }^{\mathrm{b}}$, M. Debliquy ${ }^{\mathrm{c}}$, M. Lopez-Amo ${ }^{\mathrm{a}}$, C. Caucheteur ${ }^{\mathrm{b}}$ and D. \\ Lahem $^{\mathrm{d}}$ \\ ${ }^{a}$ Universidad Pública de Navarra, Dept. of Electrical and Electronic Engineering, Campus Arrosadia \\ S/N, E-31006, Pamplona, Spain \\ ${ }^{\mathrm{b}}$ Electromagnetism and Telecommunication Department, University of Mons, Boulevard Dolez 31, \\ 7000 Mons, Belgium \\ 'Department of Materials Science, University of Mons, Rue de l'Epargne 56, 7000 Mons, Belgium \\ ${ }^{\mathrm{d}}$ Materia Nova, Materials R\&D Centre, Avenue N. Copernic 1, 7000 Mons, Belgium \\ * aitor.lopez@unavara.es; phone +34 94816 9841; fax +34948 169720
}

\begin{abstract}
In this paper, we present the results of a new $\mathrm{pH}$ sensor based on a polyaniline (PAni) coating on the surface of a tilted fiber Bragg grating. The $\mathrm{pH}$-sensitive PAni was deposited by in situ chemical oxidative polymerization. The performance of the fabricated $\mathrm{pH}$ sensor was tested and the obtained $\mathrm{pH}$ values were compared with the results obtained using a $\mathrm{pH}$ meter device. It was found that the sensor exhibits response to $\mathrm{pH}$ changes in the range of 2-12, achieving a sensitivity of $46 \mathrm{pm} / \mathrm{pH}$ with a maximum error due to the hysteresis effect of $\pm 1.14 \mathrm{pH}$. The main advantages of this PAni-TFBG pH sensor are biochemical compatibility, temperature independence, long-term stability and remote realtime multipoint sensing features. This type of sensor could be used for biochemical applications, pipeline corrosion monitoring or remote-multipoint measurements.
\end{abstract}

Keywords: optical fiber sensor, fiber Bragg grating, tilted fiber Bragg grating, Polyaniline, pH sensor, chemical sensor

\section{INTRODUCTION}

Optical and fiber-optic $\mathrm{pH}$ sensors complement the glass electrodes for $\mathrm{pH}$ measurement and offer numerous advantages such as immunity from electrical interference, feasibility of miniaturization, robustness, possibility of remote and real time sensing and in vivo measurements.

Conducting polymers possess both the mechanical properties of a polymer and the electrical properties of a conductor and have a big potential in many technological applications. Polyaniline (PAni) is a well-known conducting polymer, which is relatively easy to prepare, shows excellent stability, biochemical compatibility and has rapid and reversible adsorption or desorption kinetics [1]. It has attracted much attention in numerous sensor applications, such as gas detection [2], multi-electrode sensor arrays [3], as coatings for quartz crystal microbalance sensors [4], chemiresistors using single walled carbon nanotube (SWNT) [5] and as modified cladding in optical fiber sensors [6].

Tilted fiber Bragg grating (TFBG) sensors have proven to exhibit really interesting features when applied to chemical sensing [7]. Compared to uniform Bragg gratings (FBGs), the TFBG tilt angle causes a coupling of part of the transmitted light to the cladding of the optical fiber in the form of several cladding mode resonances [8]. Each of these modes propagates with a corresponding refractive index value and is confined in the cladding by reflection on the cladding-outer medium interface. Thus, when the refractive index value of the surrounding medium reaches a value close to the one of a mode, it loses the total internal reflection condition, being transmitted to this medium and producing a change on the grating transmitted spectrum. The monitoring of these alterations allows us to detect and quantify the variations of the refractive index of the outer medium, turning these devices into highly accurate refractometers [9].

In this work a novel $\mathrm{pH}$ sensor consisting on a polyaniline deposition on the surface of a TFBG is presented and characterized. PANI presents indeed a $\mathrm{pH}$ sensitive optical absorption spectrum in the NIR region. This effect is due to the partial protonation leading to a doping and a conformational change of the polymeric chains. The working principle of the sensor is based on the monitoring of the transmission spectrum of the TFBG, which varies with $\mathrm{pH}$ as the refractive index of the sensitive layer changes with $\mathrm{pH}$. The useful signal is a wavelength shift of the TFBG cladding modes, achieving an overall sensitivity of $46 \mathrm{pm} / \mathrm{pH}$ unit in the range 2-12 pH. Due to the main properties of this combination, PAni-TFBG pH sensors could have applications in various domains: medical smart textiles [10], 
bioelectrical and in vivo measurements [11], pipeline corrosion monitoring [12] or remote real-time multipoint for liquid solution measurements.

\section{EXPERIMENTAL SETUP AND OPERATING PRINCIPLE}

The sensor's centerpiece was a $1 \mathrm{~cm}$ long $6^{\circ}$ TFBGvphoto-inscribed in the core of a hydrogen-loaded single-mode photosensitive optical fiber (FiberCore PS-1250) using a frequency-doubled Ar laser emitting at $244 \mathrm{~nm}$ and a $1070 \mathrm{~nm}$ pitch phase mask. After the photo-inscription process, the grating remained inside an oven at $85{ }^{\circ} \mathrm{C}$ for 12 hours to get rid of the residual hydrogen content and stabilize its behavior at room temperature.

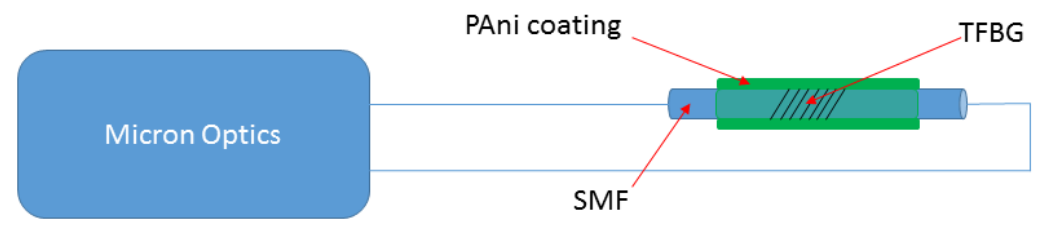

Figure 1. Experimental setup.

Fig. 1 shows the experimental setup implemented to characterize the PAni-TFBG sensing heads. Two sensors were developed to ensure the repeatability of the experiment. A commercial FBG interrogating device (Micron Optics) with a customized Labview software (scanning frequency of $10 \mathrm{~Hz}$ ) and a $5 \mathrm{pm}$ resolution was employed to analyze the sensor's optical spectrum.
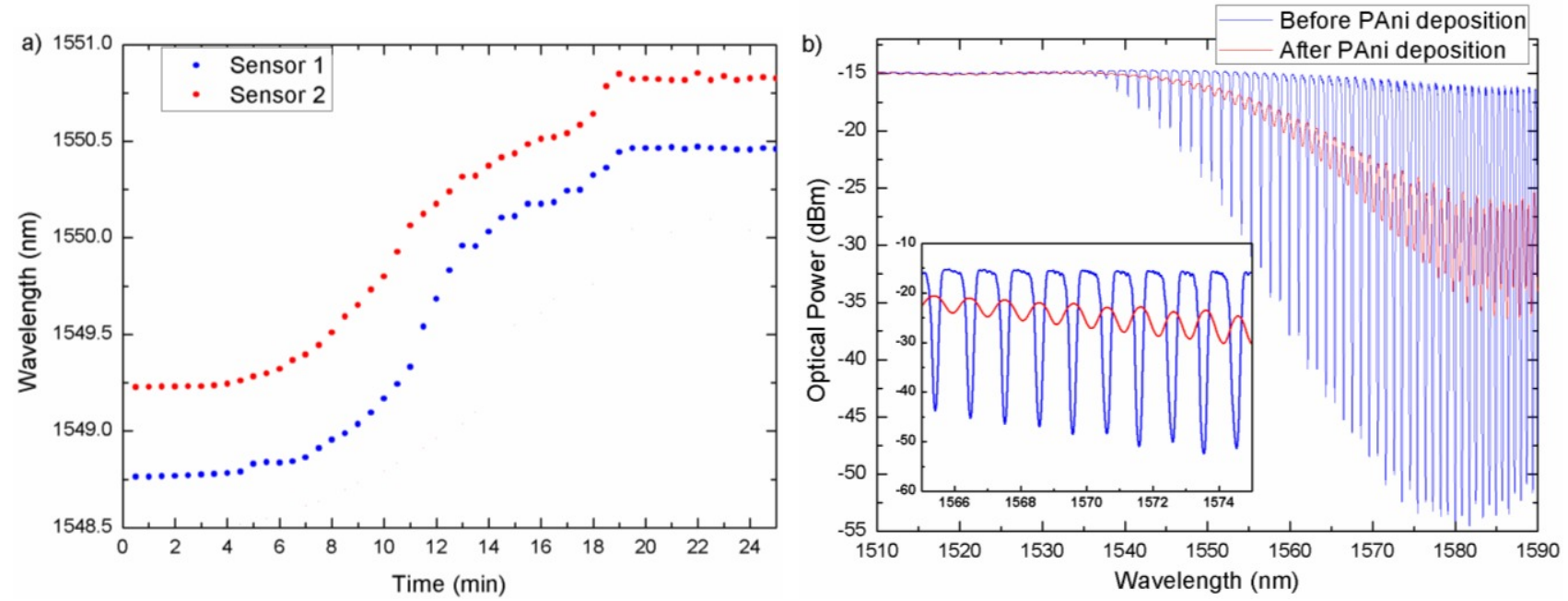

Figure 2. (a) Wavelength shift during PAni deposition and (b) optical spectrum of sensor.

PAni film was deposited by in situ oxidative polymerization during 20 minutes starting from aniline and ammonium peroxodisulfate (oxidant) in an acid aqueous solution. The sensors' optical spectrum was continuously tracked in order to find out the optimal deposition time, avoiding saturation effects, as Fig. 2b illustrates. The PAni coating modifies (rises) the external refractive index of the TFBG leading to a higher number of resonant modes coupled to the external medium and inducing a wavelength shift and an amplitude variation on the remaining cladding guided ones. The exceptional features provided by the TFBG enable direct interaction between the cladding coupled light and the bounding medium, without the need for any additional processing treatment.

\section{EXPERIMENTAL RESULTS}

The $\mathrm{pH}$ sensing heads were immersed into a $\mathrm{NaCl}$ solution at a constant temperature of $19{ }^{\circ} \mathrm{C}$ in order to perform controlled $\mathrm{pH}$ variations. $\mathrm{HCl}$ and $\mathrm{NaOH}$ were added to the $\mathrm{NaCl}$ to move towards acidic or basic aqueous solutions respectively. The $\mathrm{pH}$ was monitored with a Lab 850 (Schott Instruments) $\mathrm{pH}$ meter with a resolution of $0.001 \mathrm{pH}$. 
Notice that the refractive index of the $\mathrm{NaCl}$ solution was monitored with a refractometer (Reichert AR200) during the experiments and the refractive index of the solution is considered as negligibly affected (variations within $10^{-4}$ refractive index units (RIU) in the whole range corresponding to the resolution of the digital refractometer).

Sensor's response of one of the resonance modes is illustrated in Fig. 3.
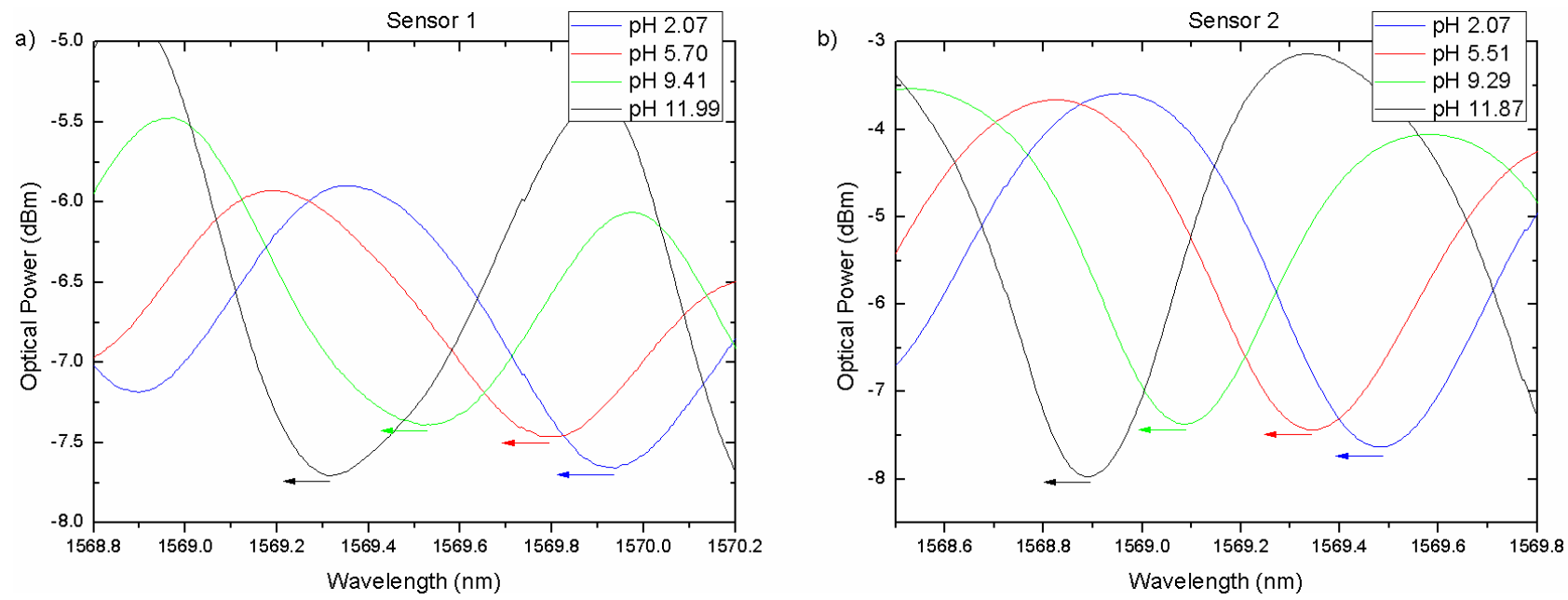

Figure 3. Wavelength shift with increasing pH: (a) Sensor 1 and (b) Sensor 2.

As can be noticed, sensor's mode resonances shifts towards lower wavelengths with increasing $\mathrm{pH}$, presenting a total shift of almost $500 \mathrm{pm}$ in a range between 2 and $12 \mathrm{pH}$. The complete responses of the sensors are shown in Fig. 4 .
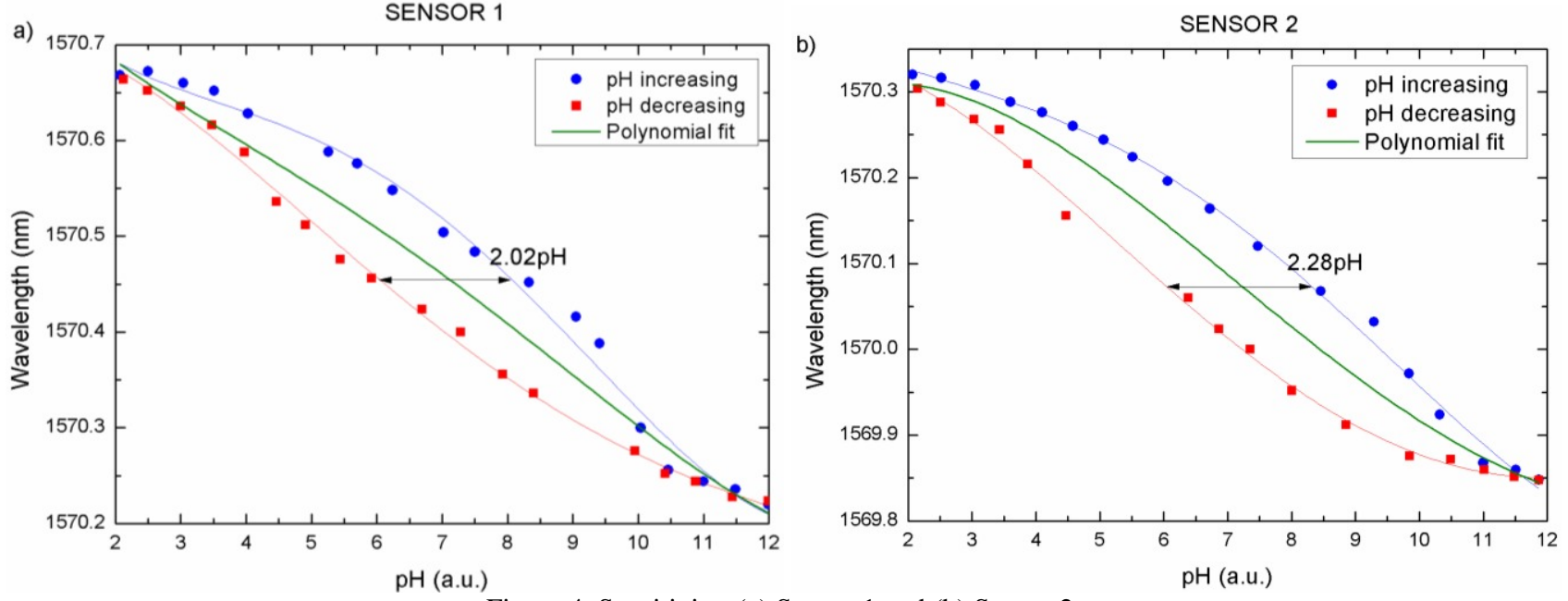

Figure 4. Sensitivity: (a) Sensor 1 and (b) Sensor 2.

On the one hand, sensors present sensitivities of $46 \mathrm{pm} / \mathrm{pH}$ and $48 \mathrm{pm} / \mathrm{pH}$ in the whole $\mathrm{pH}$ range after 3 complete cycles of $\mathrm{pH}$ to achieve stable features (slight differences are present between the first and the following cycles). On the other hand, they present an error of $\pm 1.01 \mathrm{pH}$ and $\pm 1.14 \mathrm{pH}$ respectively due to the hysteresis effect. This hysteresis, already reported for polyaniline, could be attributed to the difference in the swelling degree of polyaniline at different $\mathrm{pH}$ values which leads to a change in its porosity and conformation during various doping and de-doping processes [13]. This hysteresis can be reduced by reducing the thickness of the layer and increasing its porosity.

PAni TFBG based sensors are replicable (a high degree of similarity has been achieved between both sensors), and long term stable (sensor 1 was tested twice with a week lapse between tests). Furthermore, due to the characteristics of the TFBG structures, they are temperature independent. 


\section{CONCLUSION}

To summarize, a new fiber optic $\mathrm{pH}$ sensor has been proposed and experimentally demonstrated. The sensing head is based on a PAni coating on the surface of a TFBG. The interrogation of the sensing head has been carried out by monitoring the wavelength shift of one of the resonance modes of the TFBG. The sensor presents a sensitivity of $46 \mathrm{pm} / \mathrm{pH}$ unit in the range of $2-12 \mathrm{pH}$, which is quite broad, with a maximum error of $\pm 1.14 \mathrm{pH}$ unit due to the hysteresis effect. Two different quasi-identical sensors have been developed in order to assure the repeatability of the process. Due to the characteristics of the sensing head, it is temperature independent and biochemical compatible which make it suitable for new medical smart structures, pipeline corrosion monitoring or remote-long distance-real timemultipoint measurements.

\section{ACKNOWLEDGMENTS}

Á. González-Vila is supported by the F.R.S.-FNRS through a FRIA grant. C. Caucheteur is supported by the F.R.S.FNRS. The authors would also like to thank the financial support from the ERC (European Research Council) Starting Independent Grant PROSPER (grant agreement No. 280161 - http://www.umons.ac.be/erc-prosper), from the Spanish Comisión Interministerial de Ciencia y Tecnología within projects TEC2016-76021-C2-1-R and TEC2013-47264-C2-2$\mathrm{R}$ and from SUDOE ECOAL-MGT and FEDER funds from the European Union. The authors also acknowledge the financial support of CAPTINDOOR WBGREEN programs (Walloon Region of Belgium).

\section{REFERENCES}

[1] Chiam, Y. S., Ahad, I. Z. M., Harun, S. W., Gan, S. N. and Phang, S. W., "Effects of the Dopant Ratio on Polyaniline Coated Fiber Bragg Grating for pH detection," Synthetic Metals, 211, 132-141 (2016).

[2] Manigandan, S., Majumder, S., Ganguly, S., and Kargupta, K., "Formation of nano-rod and nano-particles of polyaniline using Langmuir-Blodgett technique," Materials Letters, 62(17), 2758-2761 (2008).

[3] Voitechovic, E., Bratov, a., Abramova, N., Razumiene, J. and Kirsanov, D., "Development of label-free impedimetric platform based on new conductive polyaniline polymer and three-dimensional interdigitated electrode array for biosensor applications," Electrochim, 173 59-66 (2015).

[4] Ihdene, Z., Mekki, A., Mettai, B., Mahmoud, R., Hamada, B., and Chehimi, M. M., "Quartz crystal microbalance VOCs sensor based on dip coated polyaniline emeraldine salt thin films," Sensors and Actuators B: Chemical, 203, 647-654 (2014).

[5] Rigoni, F., Tognolini, S., Borghetti, P., Drera, G., Pagliara, S., Goldoni, A., and Sangaletti, L., "Environmental monitoring of low-ppb ammonia concentrations based on single-wall carbon nanotube chemiresistor gas sensors: detection limits, response dynamics, and moisture effects," Procedia Engineering, 87, 716-719 (2014).

[6] Pahurkar, V. G., Tamgadge, Y. S., Gambhire, A. B., and Muley, G. G., "Evanescent wave absorption based polyaniline cladding modified fiber optic intrinsic biosensor for glucose sensing application," Measurement, 61, 9-15 (2015).

[7] Albert, J., Shao, L. Y., and Caucheteur, C., "Tilted fiber Bragg grating sensors," Laser \& Photonics Reviews, 7(1), 83-108 (2013).

[8] Lee, K. S., and Erdogan, T., "Fiber mode coupling in transmissive and reflective tilted fiber gratings," Applied optics, 39(9), 1394-1404 (2000).

[9] Laffont, G., and Ferdinand, P., "Tilted short-period fibre-Bragg-grating-induced coupling to cladding modes for accurate refractometry," Measurement Science and Technology, 12(7), 765 (2001).

[10] Massaroni, C., Saccomandi, P., and Schena, E., "Medical smart textiles based on fiber optic technology: An overview," Journal of functional biomaterials, 6(2), 204-221 (2015).

[11] Luprano, J., Sola, J., Ridolfi, A., Pasche, S., and Chetelat, O., "New generation of smart sensors for biochemical and bioelectrical applications," In Proceedings of 2007 pHealth Conference (2007).

[12] López-Higuera, J. M., Cobo, L. R., Incera, A. Q., and Cobo, A., "Fiber optic sensors in structural health monitoring," Journal of Lightwave Technology, 29(4), 587-608 (2011).

[13] Grummt, U.-W.; Pron, A.; Zagorska, M.; Lefrant, S. "Polyaniline based optical pH sensor," Anal. Chim. Acta, 357, 253-259 (1997). 